\title{
Interactions of Carbon Nanotube with Lipid Bilayer Membranes
}

\author{
Vamshi K. Gangupomu ${ }^{1}$ and Franco M. Capaldi² \\ ${ }^{1}$ Department of Chemical and Biological Engineering, Drexel University, 3141 Chestnut Street, Philadelphia, PA 19014, USA \\ ${ }^{2}$ Department of Mechanical Engineering and Mechanics, Drexel University, 3141 Chestnut Street, Philadelphia, PA 19014, USA
}

Correspondence should be addressed to Franco M. Capaldi, fmc27@drexel.edu

Received 30 September 2010; Accepted 29 January 2011

Academic Editor: Xuedong Bai

Copyright (@ 2011 V. K. Gangupomu and F. M. Capaldi. This is an open access article distributed under the Creative Commons Attribution License, which permits unrestricted use, distribution, and reproduction in any medium, provided the original work is properly cited.

Understanding the interaction between a carbon nanotube and biological macromolecules such as lipid bilayers is important for the design and development of nanovectors for gene and drug delivery. The forces of penetration and the free energies of rupture of lipid bilayers during nanotube penetration were studied using nonequilibrium, all-atom molecular dynamics simulations for pure POPC and POPC/cholesterol bilayers. The presence of cholesterol did not alter the magnitude of the rupture force and minimally increased the estimated free energy of rupture. However, the ability of the nanotube to disrupt the membrane leading to membrane poration increased with increasing cholesterol content.

\section{Introduction}

The recent growth in the field of nanomedicine resulted in a significant interest in the interactions between nanomaterials and biological structures. Carbon nanotubes (CNTs) are currently being used as atomic force microscopic probes, and there is interest in using them as nanovectors for drug delivery, gene therapy, and extracting molecules from the cells in the form of nanosyringes and nanocapsules [1-3]. The high aspect ratio and dimensions of carbon nanotubes facilitate maximum delivery of therapeutic molecules or genetic information with minimal membrane disruption. Surface functionalization of carbon nanotubes can further minimize disruption and has been proposed for targeted drug delivery to tumor sites $[4,5]$.

However, the prospect of using CNTs as nanovectors depends upon their ability to porate the cellular plasma membrane. Presently, viruses are being used as vectors for gene therapy. Viruses use various surface glycoproteins to penetrate target cell membranes thereby initiating membrane fusion ultimately delivering the genetic material [6, 7]. For example, influenza virus uses the ectodomain of fusion glycoprotein hemagglutinin (HA) to initiate fusion. The fusogenic structures of these glycoproteins are believed to expose the fusion peptide, that will eventually penetrate the target cell membrane. A similar mechanism can be used to porate cellular plasma membranes using carbon nanotubes. However, a thorough understanding of the forces involved during rupture of plasma membranes and the free energies required to rupture the membrane is important for the design and development of nanovectors.

Although pristine carbon nanotubes were previously shown to cross the cell membrane and accumulate in cytoplasmic vacuoles or the nucleus, the mechanism of internalization, however, is poorly understood. Experimental results suggested two different pathways: (1) endocytosis and (2) spontaneous insertion and diffusion across the cell membrane [8]. Prior molecular simulation studies have shown modified carbon nanotubes enter cell membrane by a lipidassisted mechanism [9]. Furthermore, Wallace and Sansom have shown that nanotubes which are oriented perpendicular to the bilayer require less force to porate the membrane than do those which are oriented either parallel or at an oblique angle to the bilayer [10]. Others have experimentally used or suggested the use of CNTs to probe the cohesive forces in a lipid bilayer [11-15].

In the present contribution we have used all-atom molecular dynamics simulations to study the forces and the change in free energy during nanotube penetration of a lipid bilayer membrane. Specifically, the free energy required to puncture the membrane and create a pore was computed. Furthermore, viruses are known to target cholesterol-rich 


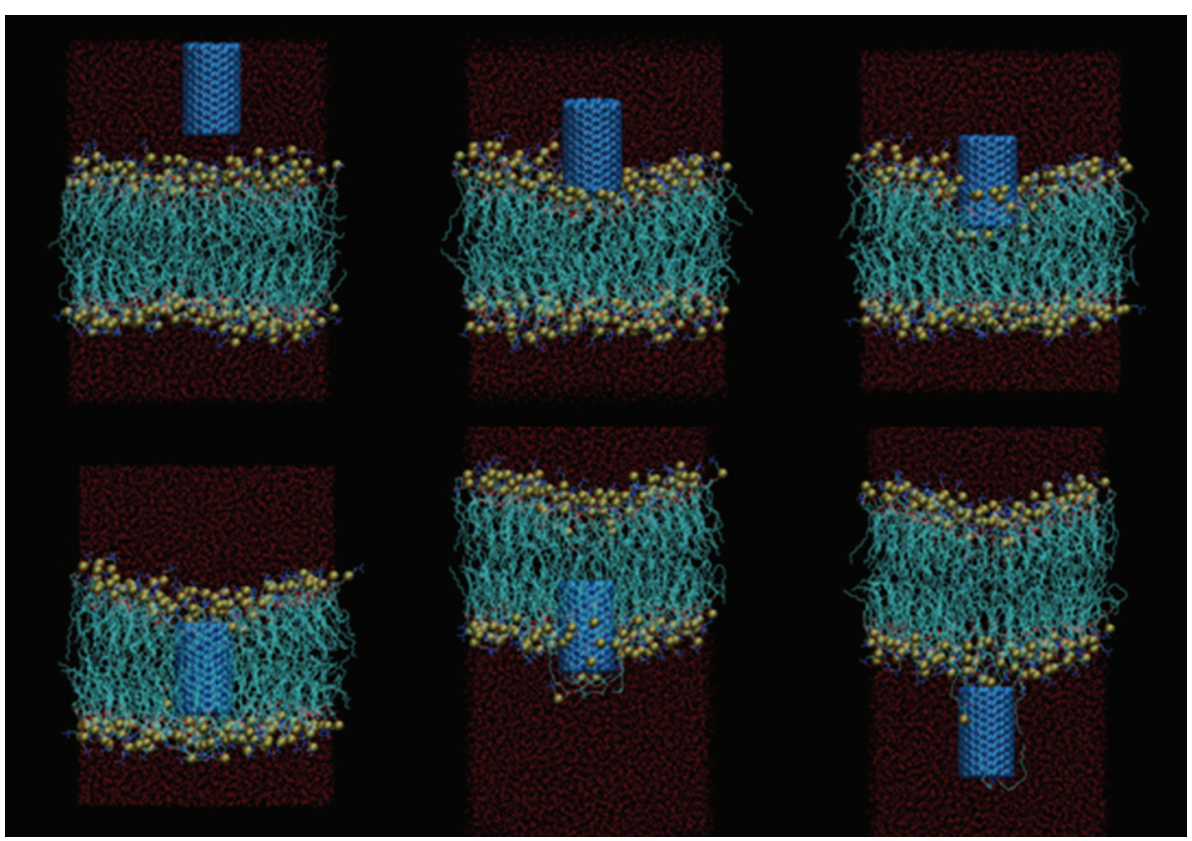

FIGURE 1: Illustration of nanotube penetrating then exiting the lipid bilayer.

domains in cell plasma membranes during infection. Therefore, the effect of cholesterol in membrane rupture and internalization process of the nanotube was also studied. We observed that the force required to porate the lipid bilayer depends on the velocity of the nanotube. However, the energy required to rupture the top monolayer and for internalization of the nanotube was $\sim 40 \mathrm{kcal} / \mathrm{mol}$ which is within the observed experimental range. Also, the addition of cholesterol to the bilayer increased the force required to porate the bilayer, but minimally increased the free energy required to rupture the bilayer. Membrane disruption due to the extraction of lipids as the CNT exits the membrane was higher in the membrane containing cholesterol.

\section{Methods}

All simulations were performed using NAMDv2.7b1 and visualized using VMD version 1.8.7 [16, 17]. The simulation system consisted of 2001-Palmitoyl,2-Oleyoyl, PhosphatidylCholine (POPC) lipids, and a carbon nanotube consisting of 400 carbon atoms. The lipid bilayer was generated using the CHARMM-GUI membrane builder and was fully hydrated with a total of $\sim 39$ water molecules per lipid using VMD [18]. Carbon nanotube of a diameter $d=10 \AA$ and a length of $\sim 20 \AA$ was later added. The CHARMM force field, with added parameters for the CNT, was employed for POPC lipids and the TIP3 water model was selected [19].

Systems were equilibrated for approximately $6 \mathrm{~ns}$ in the NPT ensemble at a constant temperature of $310 \mathrm{~K}$ and a pressure of $1 \mathrm{~atm}$ with the carbon nanotube constrained at a distance of approximately $10 \AA$ over the membrane while the membrane and surrounding water were equilibrating.
The equilibration step was followed by a simulation of membrane penetration by the CNT. Experimentally, carbon nanotubes have been observed to penetrate cellular membranes perpendicularly through a "nanospearing" effect. The forces required by the nanotube to porate a lipid bilayer have been observed to be the smallest when the nanotube was oriented perpendicular rather than oblique or parallel to the bilayer. Therefore, we constrained the lateral degrees of freedom and studied the forces and the change in the freeenergy profile as the nanotube penetrates the lipid bilayer to understand the internalization pathway, Figure 1. Constant velocity Steered Molecular Dynamics (cv-SMD) was used to simulate this step. The CNT was moved using a dummy atom that is attached to the center of mass of the nanotube via a virtual spring with a spring constant, $k$, of $7 \mathrm{kcal} / \mathrm{mol} / \AA^{2}$. This spring constant was selected to maintain a stiff spring [20]. The dummy atom was moved at a constant velocity in the direction perpendicular to the membrane plane. The lateral motion of the dummy atom was constrained with a force constant of $10 \mathrm{kcal} / \mathrm{mol} \AA$. The constraint forces can be calculated from the displacement

$$
\begin{gathered}
F=-\nabla U, \\
U=\frac{1}{2} k\left(v t-\left(\vec{r}-\vec{r}_{o}\right) \cdot \vec{n}\right)^{2},
\end{gathered}
$$

where $k$ is the spring constant, $t$ is the current time, $\vec{r}$ is the position of the dummy atom, $\vec{r}_{o}$ is the initial position of the dummy atom, and $v$ is the velocity at which the dummy atom is moved. 


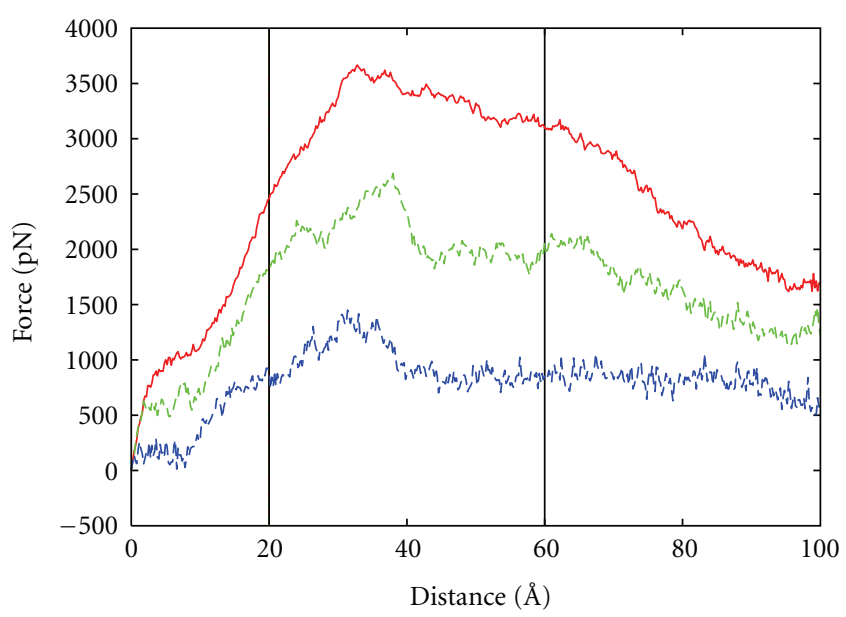

Figure 2: The force distance profiles of nanotube penetration at $v=0.1 \AA / \mathrm{ps}$ (red), $v=0.05 \AA / \mathrm{ps}$ (green), and $0.01 \AA / \mathrm{ps}$ (blue). The location of the edges of the membrane are shown as vertical lines at $20 \AA$ and $40 \AA$.

The potential of mean force (PMF) was determined using the Jarzynki's equation, that was generalized for the isobaricisothermal systems $[20,21]$

$$
e^{-\beta \Delta G}=\left\langle e^{-\beta W}\right\rangle
$$

where $\beta=1 / k_{B} T, k_{B}$ is the Boltzmann Constant, $T$ is the temperature, $G$ is the Gibbs free energy, and $W$ is the work done. The work done along the reaction coordinate path was calculated as the integral of the force on the spring along the direction of steering. The second-order cumulant of Jarzynski's equation, that is valid with the use of a sufficiently stiff spring, was used to compute the PMF.

For the cholesterol simulations, a lipid bilayer consisting of 200 lipid molecules with 70\% POPC and 30\% cholesterol was generated using CHARMM-GUI membrane builder. After the addition of CNT, the membrane was equilibrated for approximately $6 \mathrm{~ns}$. The SMD and PMF calculations were performed as described for the pure POPC bilayer.

\section{Results}

3.1. Force-Distance Profiles. CNT insertion was performed at three velocies $v=0.1,0.05$, and $0.01 \AA / p s$ using cv$\mathrm{SMD}$, and the forces required to pull the nanotube were calculated using (2). The force was plotted as a function of the distance travelled by the dummy atom along the $z$ axis as the nanotube traversed the membrane, Figure 2. The magnitude and the profiles of the force-distance curves depended upon the CNT velocity. After an initial acceleration, the nanotube reached a constant force in water. This constant force is more pronounced at the lowest pulling speed at approximately $50 \mathrm{pN}$. A second phase consisting of increasing force followed, which can probably be attributed to the increasing membrane curvature. As the nanotube approches the lipid bilayer, a curvature defect is created in the membrane. However, for the nanotube to penetrate the lipid bilayer, it must create a hydrophobic defect in the bilayer exposing the hydrophobic tail groups to water. The energy required to porate the membrane depends on the interplay between the curvature and hydrophobic defects of the membrane [22]. Previous coarse-grained simulations have shown that as the nanotube approaches the lipid bilayer the applied force becomes negative [10]. However, it should also be noted that the velocity of nanotube penetration in this study is higher and induces a negative curvature in the lipid bilayer. Therefore, the mechanism of nanotube penetration in to the exoplasmic monolayer probably depends on the velocity of CNT. At lower velocity, the lipid head groups have more time for lateral movement and let the nanotube in to the membrane core and relieve the hydrophobic mismatch. This might also explain the higher forces of penetration predicted in simulations compared to experiments. Similarly, the penetration force was also observed to increase with velocity in our simulations. In all cases, the highest force was observed as the nanotube separated the lipid head groups. This phase was followed by a decrease in the applied force as the nanotube entered the hydrophobic core of the membrane. After the nanotube entered the membrane, the hydrophobic mismatch between the lipid molecules and the nanotube resulted in the lipid tails wrapping around the outer surface of the nanotube to reduce unfavorable exposure of the hydrophobic core to water thereby reducing the pulling forces. The hydrophobic amino acid residues on virus fusion peptides make use of this to anchor in the target cell membrane. A second peak could be observed between $60 \AA$ and $70 \AA$ as the nanotube exits the membrane at a velocity of $0.05 \AA / \mathrm{ps}$ as it penetrates the head groups of the lower lipid monolayer. However, at the highest velocity of $0.1 \AA /$ ps, although a second peak can be observed, the force required for penetration is very high and very little membrane deformation was observed. At the lowest velocity of $0.01 \AA / \mathrm{ps}$, the lipid molecules had time to adopt favorable configurations on the surface of the CNT and some were removed along with the nanotube. This blunted the second force peak since a portion of the membrane was pulled out with the CNT. Blocking of nanotube by lipids, especially those from the lower leaflet, was previously observed in simulation studies [10]. Therefore, poration forces depend on the energies involved in membrane bending, breaking of the hydrogen bonds between the head groups and water molecules, the hydrophilic interactions between the lipid head groups, the hydrophobic interactions between the nanotube and the lipids, and the energy required for the lateral movement of the lipid molecules. The fricton between the nanotube and the membrane lipids also plays an important role in the total force required for membrane penetration.

To estimate the effect of cholesterol on the forces required for nanotube penetration, we compared simulations of pure POPC with a lipid bilayer containing 70\% POPC and 30\% cholesterol using a CNT penetration velocity of $0.01 \AA / \mathrm{ps}$. A comparison of the force-distance curve with that of pure POPC bilayers (Figure 3 ) showed a reduction in membrane bending indicating the membrane stiffness had increased. However, the force required to puncture the membrane 


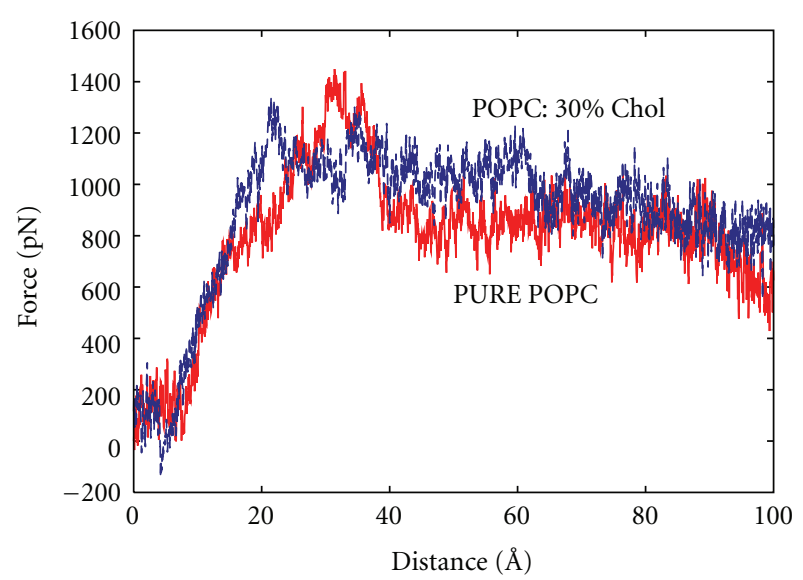

FIgURE 3: Force-Distance profile comparison for pure POPC (red) and POPC/cholesterol (blue) bilayers.

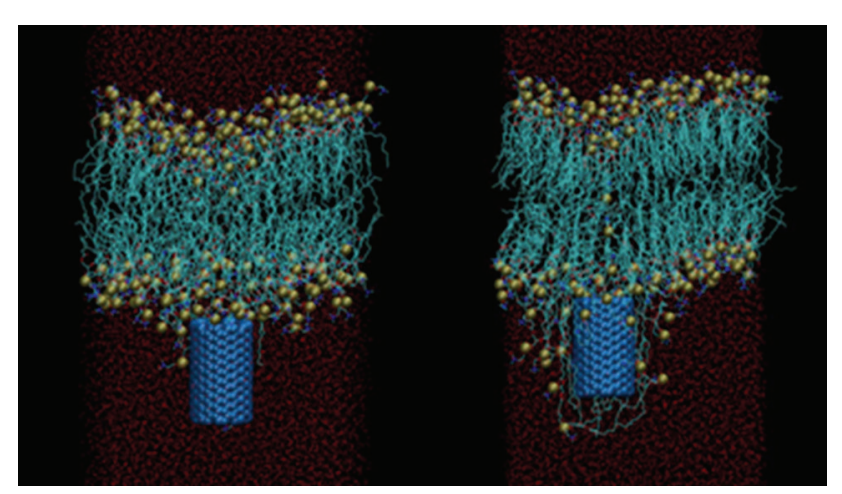

Figure 4: Disruption of lipid bilayer by carbon nanotube for pure POPC (left) and POPC/cholesterol (right).

remained similar to the pure POPC bilayers. Although the presence of cholesterol stabilizes the bilayer, cholesterol also partitions into the spaces between the lipid molecules and therefore induces a spontaneous negative curvature of the bilayer. Further, a second peak was observed as the nanotube exits the bilayer and the force required for the nanotube to exit the bilayer was also higher than pure POPC. It was also observed that as the nanotube exits the bilayer, a higher number of lipids were extracted from the bilayer containing $30 \%$ cholesterol than from the pure POPC (Figure 4).

3.2. Potential of Mean Force (PMF). The free energy barrier required for the carbon nanotube to penetrate the cell membrane was computed. A curvature defect was observed in the membrane as the nanotube approaches the membrane. By creating a hydrophobic defect, exposing the hydrophobic membrane core to water, the CNT is incorporated into the membrane. CNT incorporation is accompanied by the breaking of Hydrogen bonds between lipid head groups and water and between the lipid molecules themselves. Spearing would reduce this energy barrier, and the nanotube can be internalized with minimal membrane disruption. The PMF was plotted with respect to the distance travelled by

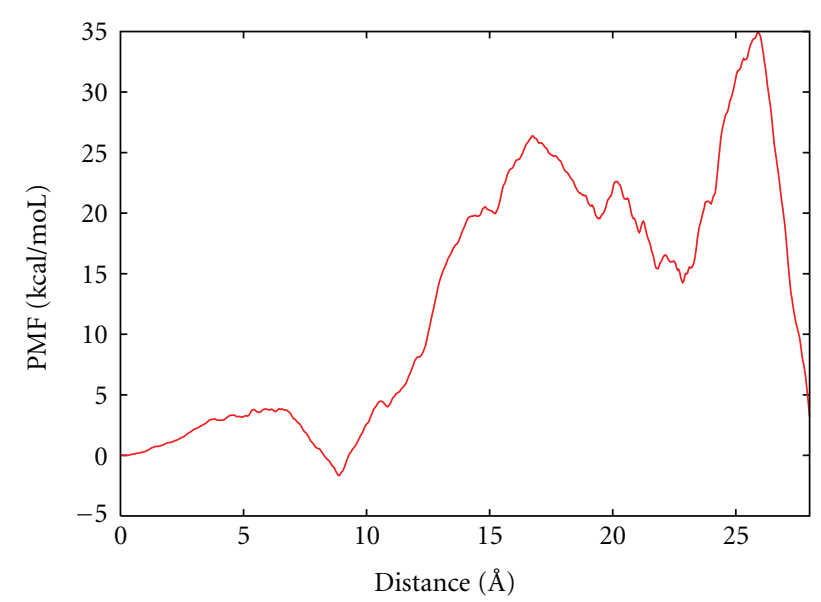

(a)

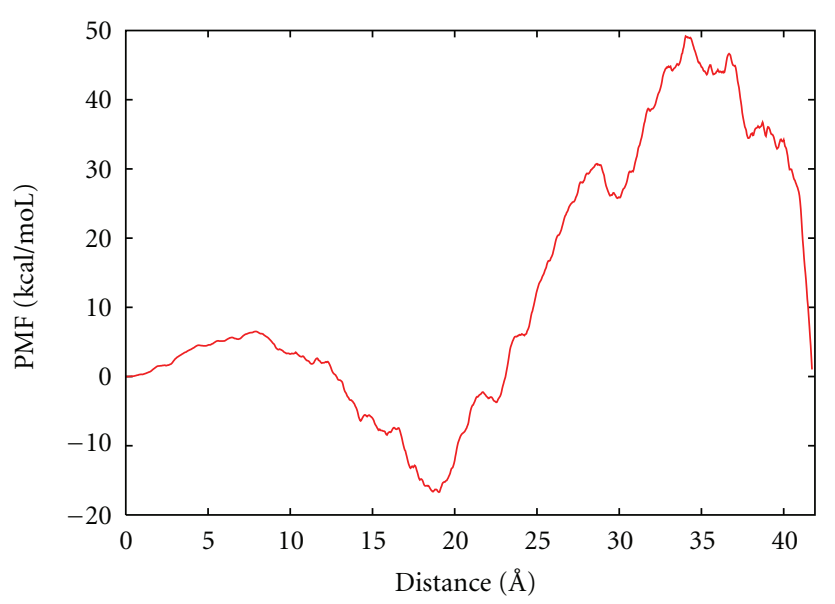

(b)

FIGURE 5: Potential of mean force for a CNT penetrating (a) a POPC bilayer and (b) a POPC bilayer with cholesterol.

the dummy atom. The work done to pull the nanotube across the lipid bilayer using the $\mathrm{cv}$-SMD simulations was used to calculate the potential of mean force. However, for Jarzynski's equality to be applicable, the transformation should be reversible. Therefore, the PMF for the pure lipid bilayer was calculated at only the two lower velocities of $0.05 \AA /$ ps (data not shown) and $0.01 \AA /$ ps. Following membrane rupture, the nanotube disrupts the membrane structure irreversibly by dragging lipid molecules along with it. Therefore, the PMF was computed prior to the irreversible disruption events. Furthermore, the PMF peaks as the nanotube approaches the membrane and is followed by a decrease in free energy as the nanotube breaches the lipid head groups.

The highest energy barrier of $\sim 35 \mathrm{kcal} / \mathrm{mol}$ was observed for nanotube velocity of $0.01 \AA /$ ps in pure POPC (Figure 5), as the nanotube porates the top monolayer of the membrane. This energy is on the order of the energy required to porate a lipid monolayer experimentally which was expected to be $\sim 40-55 \mathrm{kcal} / \mathrm{mol}$. The energy released in relieving the membrane curvature defect facilitates nanotube internalization and the membrane poration. After the nanotube 
crosses the energy barrier, the nanotube is spontaneously internalized by the hydrophobic lipid tails and the PMF decreases. Following this phase the PMF should be expected to have a minimum at the center of the bilayer. The free energy of rupture at $0.05 \AA /$ ps was also within the expected experimental range.

The addition of $30 \%$ cholesterol increased the free energy of rupture to approximately $50 \mathrm{kcal} / \mathrm{mol}$. Furthermore, the energy maxima for the pure POPC bilayer occurs when the CNT breaks hydrogen bonds between the head groups. However, the maxima is observed in the cholesterol-containing bilayer when the fused ring structure of the cholesterol is disrupted. The formation of the two-three extended coiled coils required to porate a cell membrane by viruses has been estimated to provide $\sim 60-90 \mathrm{kcal} / \mathrm{mol}$ of energy [22]. However, it should be noted that the amino acid sequence of the viral fusion peptides and the composition of the lipid bilayers also play an important role in determining the forces required and the energetics of membrane rupture during viral infection. In the absence of an external applied force on the nanotube, there would probably be minimal membrane bending and the mechanism of internalization would depend only on the hydrophobic mismatch. This would explain the spontaneous internalization of carbon nanotube with small diameters. However, for the nanotube to traverse the bilayer, a two-phase internalization mechanism involving an energy requiring step is needed to cross the saddle point at endoplasmic head groups.

\section{Conclusion}

We used nonequilibrium all-atom molecular dynamic simulations to study the forces required to puncture and the free energy of rupture of pure POPC and POPC/cholesterol bilayers with a carbon nanotube. While the observed penetration forces were higher than those observed experimentally, the rupture force depends on the velocity of nanotube pentration. Decreasing the speed decreases the penetration force as observed both during our simulations and previous coarsegrained simulations. The free energies required to rupture a bilayer with a nanotube were also computed. However, the observed rupture energies were within the estimates of rupture by an electric pulse [22]

The presence of $30 \%$ cholesterol did not significantly alter the rupture forces or free energies. However, the bilayer containing cholesterol had an observable second peak in the force-distance profiles. This suggests that the presence of cholesterol would help to achor the nanotube once it has overcome the initial barrier. This would probably explain the prefential partioning of viral fusion peptides in cholesterol-rich domains. The presence of cholesterol stabilizes the bilayer helping to anchor the viral surface proteins prior to fusion. Furthermore, during fusion the presence of cholesterol promotes membrane disruption. Previous experimental results have also shown increased transfer of aqueous contents between cells expressing influenza HA and red blood cells with increasing cholesterol content. It was therefore hypothesized that cholesterol promotes pore widening. Our results also suggest that the presence of cholesterol promotes formation of larger membrane pores. It should however be noted that the presence of a variety of lipid molecules and the side chains in viral proteins might alter the mechanism of internalization of surface proteins and free energies of rupture.

\section{Acknowledgment}

This research was in part supported by Grant no. MCB080010P from the Pittsburgh Supercomputing Center, supported by several federal agencies, the Commonwealth of Pennsylvania and private industry.

\section{References}

[1] A. Bianco and M. Prato, "Can carbon nanotubes be considered useful tools for biological applications?” Advanced Materials, vol. 15, no. 20, pp. 1765-1768, 2003.

[2] D. Cai, J. M. Mataraza, Z. H. Qin et al., "Highly efficient molecular delivery into mammalian cells using carbon nanotube spearing," Nature Methods, vol. 2, no. 6, pp. 449-454, 2005.

[3] L. Monticelli, E. Salonen, P. C. Ke, and I. Vattulainen, "Effects of carbon nanoparticles on lipid membranes: a molecular simulation perspective," Soft Matter, vol. 5, no. 22, pp. 44334445, 2009.

[4] A. Bianco, K. Kostarelos, C. D. Partidos, and M. Prato, "Biomedical applications of functionalised carbon nanotubes," Chemical Communications, no. 5, pp. 571-577, 2005.

[5] A. Bianco, K. Kostarelos, and M. Prato, "Applications of carbon nanotubes in drug delivery," Current Opinion in Chemical Biology, vol. 9, no. 6, pp. 674-679, 2005.

[6] D. M. Eckert and P. S. Kim, "Mechanisms of viral membrane fusion and its inhibition," Annual Review of Biochemistry, vol. 70, pp. 777-810, 2001.

[7] P. L. Yeagle, Ed., The Structure of Biological Membranes, CRC Press LLC, 2005.

[8] X. Shi, Y. Kong, and H. Gao, "Coarse grained molecular dynamics and theoretical studies of carbon nanotubes entering cell membrane," Acta Mechanica Sinica, vol. 24, no. 2, pp. 161-169, 2008.

[9] C. F. Lopez, S. O. Nielsen, P. B. Moore, and M. L. Klein, "Understanding nature's design for a nanosyringe," Proceedings of the National Academy of Sciences of the United States of America, vol. 101, no. 13, pp. 4431-4434, 2004.

[10] E. J. Wallace and M. S. P. Sansom, "Blocking of carbon nanotube based nanoinjectors by lipids: a simulation study," Nano Letters, vol. 8, no. 9, pp. 2751-2756, 2008.

[11] V. Barwich, M. Bammerlin, A. Baratoff et al., "Carbon nanotubes as tips in non-contact SFM," Applied Surface Science, vol. 157, no. 4, pp. 269-273, 2000.

[12] L. Chen, C. L. Cheung, P. D. Ashby, and C. M. Lieber, "Singlewalled carbon nanotube AFM probes: optimal imaging resolution of nanoclusters and biomolecules in ambient and fluid environments," Nano Letters, vol. 4, no. 9, pp. 1725-1731, 2004.

[13] H. Dai, J. H. Hafner, A. G. Rinzler, D. T. Colbert, and R. E. Smalley, "Nanotubes as nanoprobes in scanning probe microscopy," Nature, vol. 384, no. 6605, pp. 147-150, 1996. 
[14] F. Z. Fang, Z. W. Xu, S. Dong, and G. X. Zhang, "High aspect ratio nanometrology using carbon nanotube probes in atomic force microscopy," Cirp Annals-Manufacturing Technology, vol. 56, no. 1, pp. 533-536, 2007.

[15] I. Obataya, C. Nakamura, S. Han, N. Nakamura, and J. Miyake, "Nanoscale operation of a living cell using an atomic force microscope with a nanoneedle," Nano Letters, vol. 5, no. 1, pp. 27-30, 2005.

[16] W. Humphrey, A. Dalke, and K. Schulten, "VMD: visual molecular dynamics," Journal of Molecular Graphics, vol. 14, no. 1, pp. 33-38, 1996.

[17] L. Kalé, R. Skeel, M. Bhandarkar et al., "NAMD2: greater scalability for parallel molecular dynamics," Journal of Computational Physics, vol. 151, no. 1, pp. 283-312, 1999.

[18] S. Jo, J. B. Lim, J. B. Klauda, and W. Im, "CHARMM-GUI membrane builder for mixed bilayers and its application to yeast membranes," Biophysical Journal, vol. 97, no. 1, pp. 5058, 2009.

[19] S. E. Feller and A. D. MacKerell, "An improved empirical potential energy function for molecular simulations of phospholipids," Journal of Physical Chemistry B, vol. 104, no. 31, pp. 7510-7515, 2000.

[20] S. Park, F. Khalili-Araghi, E. Tajkhorshid, and K. Schulten, "Free energy calculation from steered molecular dynamics simulations using Jarzynski's equality," Journal of Chemical Physics, vol. 119, no. 6, pp. 3559-3566, 2003.

[21] S. Park and K. Schulten, "Calculating potentials of mean force from steered molecular dynamics simulations," Journal of Chemical Physics, vol. 120, no. 13, pp. 5946-5961, 2004.

[22] J. Bentz, "Membrane fusion mediated by coiled coils: a hypothesis," Biophysical Journal, vol. 78, no. 2, pp. 886-900, 2000. 

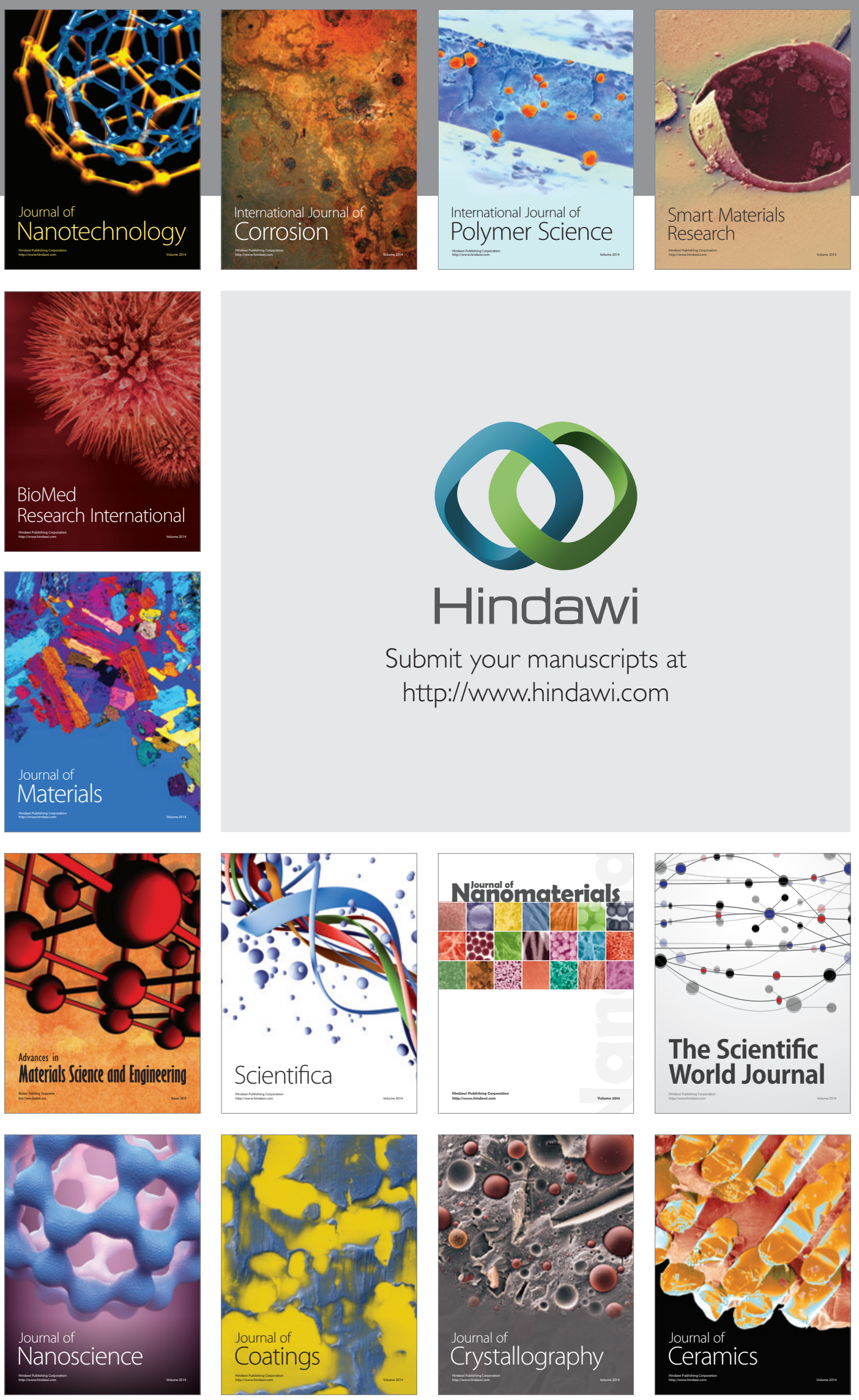

The Scientific World Journal

Submit your manuscripts at

http://www.hindawi.com

\section{World Journal}

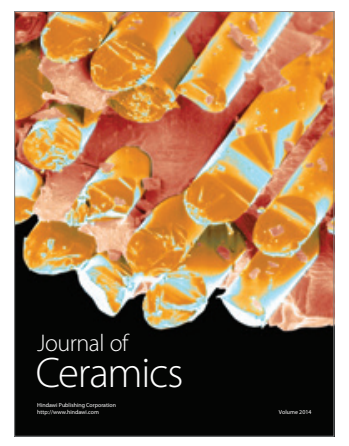

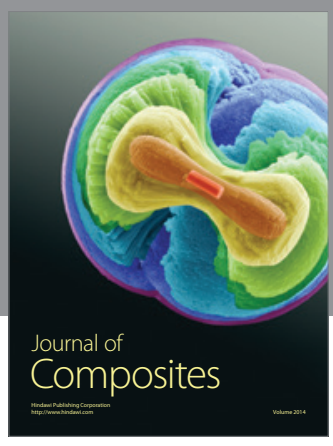
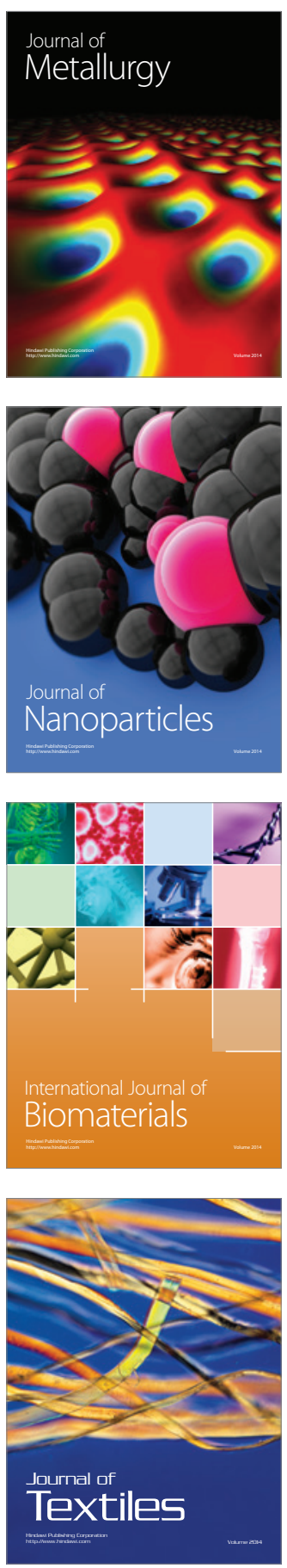